\title{
POPULATION STUDY ARTICLE Erythropoietin serum levels, versus anaemia as risk factors for severe retinopathy of prematurity
}

\author{
Pia Lundgren ${ }^{1}$, Gunnel Hellgren ${ }^{1}$, Aldina Pivodic ${ }^{2}$, Karin Sävman ${ }^{3}$, Lois E. H. Smith ${ }^{4}$ and Ann Hellström ${ }^{1}$
}

BACKGROUND: Preterm infants with anaemia are treated with recombinant human erythropoietin (rhEPO). It is debated whether rhEPO treatment is a risk factor for retinopathy of prematurity (ROP). We evaluated longitudinal EPO and haemoglobin levels, blood transfusions and neonatal morbidities as risk factors for severe ROP.

METHOD: This prospective study included 78 Swedish infants, born $<28$ weeks gestational age (GA), screened for ROP. We tested serum EPO levels on postnatal days 1, 7, 14 and 28 and at postmenstrual ages 32, 36 and 40 weeks. Haemoglobin levels and blood transfusions were recorded during postnatal weeks $1-4$. Anaemia was defined as haemoglobin $\leq 110 \mathrm{~g} / \mathrm{L}$.

RESULTS: During postnatal week 1 , infants with severe ROP requiring treatment $(28 \%)$ more frequently developed anaemia $(42.9 \%$ versus $8.0 \%, P=0.003$ ) and had higher mean EPO levels (postnatal day $7: 14.2$ versus $10.8 \mathrm{mlU} / \mathrm{mL}, P=0.003$ ) compared to infants with no or less severe ROP not requiring treatment. In multivariable analyses, GA and anaemia during week 1 remained significant risk factors, but elevated EPO level postnatal day 7 was no longer significant.

CONCLUSIONS: Among infants born $<28$ weeks GA, anaemia during week 1 was a significant risk factor for severe ROP requiring treatment but not elevated EPO levels.

Pediatric Research (2019) 86:276-282; https://doi.org/10.1038/s41390-018-0186-6

\section{INTRODUCTION}

Erythropoietin (EPO) is an oxygen-regulated haematopoietic growth factor that promotes erythrocyte formation (erythropoiesis) in bone marrow. EPO production is stimulated by hypoxia and anaemia, and it is inhibited by proinflammatory cytokines. ${ }^{1} \mathrm{~A}$ major action of EPO is to maintain haemoglobin levels constant for optimal tissue oxygen saturation by stimulating haemoglobin synthesis, particularly after haemorrhage. However, EPO was also shown to provide major tissue-protective functions in the central nervous system, heart, kidney, liver and vasculature. ${ }^{2,3}$

Anaemia of prematurity is a common condition in preterm infants, particularly in extremely preterm infants, born before 28 weeks of gestation. ${ }^{4}$ In these conditions, anaemia is caused by an immature haematopoietic system, inadequate EPO production and iatrogenic blood loss, due to frequent blood sampling. ${ }^{5}$ Most extremely preterm infants receive blood transfusions for anaemia at some point during hospitalization, ${ }^{6}$ based on haemoglobin levels and clinical indications, including oxygen requirements. ${ }^{7}$ Worldwide, many neonatal intensive care units use recombinant human erythropoietin (rhEPO) to treat anaemia of prematurity. ${ }^{8}$

Retinopathy of prematurity (ROP) is a sight-threatening complication of preterm birth that affects the developing retina. ${ }^{9}$ ROP presents in two phases. Phase 1 starts at a preterm birth and persists during the infants' first weeks of life. In phase 1, hyperoxia suppresses the production of growth factors, such as vascular endothelial growth factor (VEGF) and EPO. The lack of growth factors delays normal retinal vessel growth and leads to retinal hypoxia. Phase 2 ROP develops weeks to months after birth, starting around 30 weeks postmenstrual age (PMA). At that time, the incompletely vascularized neural retina matures, and its metabolic demands increase. The hypoxic avascular retina stimulates large increases in VEGF and EPO, which results in uncontrolled retinal neovascularization in phase $2 .^{10}$ Without timely treatment, neovascularisation can lead to retinal detachment and blindness. ROP pathogenesis is multifactorial, and ROP risk factors may be phase-dependent. ${ }^{11}$

In preterm infants, vitreous levels of EPO were elevated in infants that developed neovascularization during phase 2 ROP. ${ }^{12}$ Moreover, serum EPO levels were elevated on postnatal day 14 , in preterm infants that developed severe $\mathrm{ROP}^{13,14}$ and reduced on postnatal day 28 in preterm infants that developed any degree of ROP. ${ }^{15}$ Consequently, it remains unclear whether rhEPO treatment is a risk factor for ROP. ${ }^{16-23}$ Some have suggested that the timing of rhEPO treatment is crucial. ${ }^{23,24}$

No previous study has investigated the correlations between serum EPO levels, anaemia, blood transfusions and the development of ROP in preterm infants. These associations need to be elucidated further.

In this study, we evaluated the longitudinal relationship between serum EPO levels and anaemia, blood transfusions and ROP requiring treatment in preterm infants.

\section{METHODS}

We included infants with gestational age (GA) $<28$ weeks admitted to the neonatal intensive care unit at Sahlgrenska University Hospital in Gothenburg, Sweden, from 4 April 2013 to 22 September 2015. Exclusion criteria were any major congenital

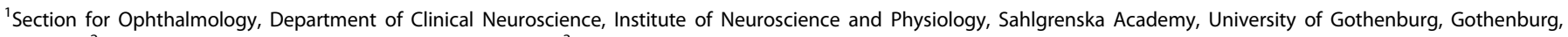

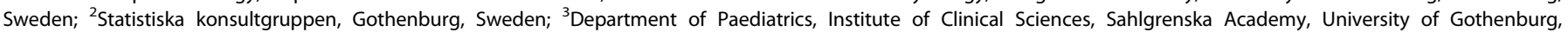
Gothenburg, Sweden and ${ }^{4}$ The Department of Ophthalmology, Boston Children's Hospital, Harvard Medical School, Boston, MA, USA Correspondence: Pia Lundgren (pia.lundgren@gu.se) 
malformation or death before completing ROP screening (approximately 40 weeks PMA). ${ }^{25}$

Birth characteristics and neonatal infant morbidities

We recorded the birth characteristics of all infants, including sex, GA and birth weight (BW). We also recorded neonatal morbidities up to 40 weeks PMA, including sepsis, bronchopulmonary dysplasia (BPD), intraventricular haemorrhage (IVH) and necrotizing enterocolitis (NEC). Sepsis was defined as a positive response in blood cultures, or it was based on clinical symptoms and signs plus increased serum levels of C-reactive protein. BPD was defined as the need for supplemental oxygen at PMA 36 weeks. IVH was recorded in cases involving any grade of $\mathrm{IVH}$; severe IVH was defined as $I V H \geq$ grade 3 . NEC was defined according to Bell's staging system. ${ }^{26}$

ROP examinations were performed according to a routine protocol that consisted of dilated ocular fundus examinations. ROP was classified with the International Classification of Retinopathy of Prematurity. ${ }^{27}$ Treatment was provided according to recommendations of the Early Treatment for Retinopathy of Prematurity Cooperative Group. ${ }^{28}$

Blood sampling and analyses of erythropoietin and haemoglobin For EPO, blood samples were collected on postnatal days $1,7,14$ and 28, and at PMA 32, 36 and 40 weeks, according to the study protocol. Samples were centrifuged, aliquoted and placed in $-70^{\circ}$ $\mathrm{C}$ within $2 \mathrm{~h}$ after blood sampling. Serum concentrations of EPO were analysed using ELLA multi-analyte platform (Biotechne, San José, CA, USA) according to the protocol provided by the manufacturer. Briefly, $50 \mu \mathrm{l}$ sample, diluted 1:4 in SD 13 buffer, was applied in cartridge wells, and then the atomized analysis procedure was finalized within $70 \mathrm{~min}$. Raw data were processed by the Simple Plex Explorer Software. Inter-assay coefficients of variation (CVs) tested between 40 assays were $7.41 \%, 5.51 \%$ and $4.01 \%$ at $2.8,14.6$ and $142.9 \mathrm{mIU} / \mathrm{mL}$, respectively. Intra-assay CV based on nine measurements was $6.39 \%$ at $14.7 \mathrm{mlU} / \mathrm{mL}$.

For haemoglobin, routine blood samples were drawn according to local guidelines and clinical indications. All available values for haemoglobin levels were retrieved during the infants' first 4 weeks of life. For each infant, when multiple haemoglobin values were available for the same day, we calculated the mean haemoglobin based on the minimum and maximum haemoglobin for that day. We averaged the daily haemoglobin values to calculate the mean weekly haemoglobin for each infant. Anaemia was defined as haemoglobin $\leq 110 \mathrm{~g} / \mathrm{L}$. The lowest haemoglobin value per day was evaluated to determine whether anaemia had occurred that day. One or more days of anaemia during the week was considered a week with anaemia. The dates and numbers of blood transfusions were recorded. Decisions concerning blood transfusions were based on local guidelines and clinical indications. The proportion of patients requiring blood transfusion was analysed longitudinally. We analysed whether the weekly average amount of blood delivered per day was associated with severe ROP.

Risk stratification

We assessed the impact of risk factors in different time periods, as follows: the first postnatal day (0-1 days); the first postnatal week (0-7 days); the second postnatal week (8-14 days); and the third through fourth postnatal weeks (15-28 days).

Statistical analyses

Descriptively, categorical variables were expressed as the number and percentage. Continuous variables were expressed as the median and interquartile range or the mean and standard deviation (SD), as applicable. Comparisons between two groups were performed with Fisher's exact test for dichotomous variables and the Mann-Whitney $U$ test for continuous variables.
Longitudinal data on EPO levels and haemoglobin levels were expressed as the mean level and $95 \%$ confidence interval $(\mathrm{Cl})$. The means were evaluated for a typical patient with ROP that did or did not require treatment, by constructing random coefficient models with unstructured covariance patterns, and underlying cubic splines, to account for nonlinear changes over time. We found that a Gaussian distribution, including an empirical bias correction, was satisfactory, based on residual plots. EPO was analysed on a natural log scale and back-transformed for graphical representations, that is, geometric means were presented instead of arithmetic ones. The probability of experiencing anaemia and requiring blood transfusion were analysed with similar methods, except the model assumed a binomial distribution and applied a quadrature method. Analyses were performed with the SAS Software procedure, PROC GLIMMIX.

We used a univariable and GA-adjusted logistic regression model to analyse the ability to predict the development of severe ROP (in each time period mentioned above), based on the levels of EPO, haemoglobin (mean/day) or anaemia (any day/week) and the mean amount of blood transfused. We investigated the nonlinear relationship between these variables and the development of severe ROP with piecewise linear functions. The linear or the piecewise linear result with the lowest Akaike's information criterion was selected to represent the effect of a given variable. The area under the receiver operating characteristic (ROC) curve was taken as a goodness-offit statistic. Multivariable logistic models, adjusting for anaemia, blood transfusion and anaemia, together with GA, were used to evaluate the ability of EPO to predict severe ROP. Firth's penalized maximum likelihood estimation was applied to reduce bias in the parameter estimates for cases of partial or complete separation, leading to non-estimable coefficients or $95 \% \mathrm{Cls}$. The effect of EPO on treated ROP was shown in a probability plot obtained from the logistic regression. The observed proportions of infants with treated ROP with exact $95 \% \mathrm{Cls}$ were included in the same figure in order to evaluate the calibration property of the model. ${ }^{29}$

All tests were two-tailed and conducted at 0.05 significance level. All analyses are performed with the SAS Software, version 9.4 (SAS Institute Inc., Cary, NC, USA).

\section{RESULTS}

Clinical characteristics and postnatal morbidities

Of the 138 infants born at GA $<28$ weeks during the study period, 90 eligible infants were enroled after receiving parental informed consent to participate. Twelve infants died before 40 weeks PMA. Therefore, we analysed data on 78 infants. The mean GA was 25.5 (SD 1.4) weeks and the mean BW was 797 (SD 223) g. A total of 22/ $78(28 \%)$ infants developed severe ROP, which required treatment. These infants were more immature at birth (mean (SD): 24.5 (1.0) weeks versus $26.0(1.3)$ weeks $G A, P<0.001)$ and had a lower mean BW $658(160) \mathrm{g}$ versus $852(222) \mathrm{g}, P<0.001)$ compared to infants that did not require ROP treatment (Table 1). The birth characteristics, postnatal morbidities and explanatory variables are shown in Table 1.

Association between longitudinal serum EPO and ROP treatment We examined longitudinal levels of EPO from birth to 40 weeks PMA over 150 postnatal days, among infants that require ROP treatment compared to those that did not require treatment (Fig. 1a). Fig. $1 \mathrm{~b}$ presents a more detailed graph of the first 28 postnatal days. We found that the mean EPO levels on postnatal day 7 were higher in infants that required ROP treatment $(14.2$, SD $13.2 \mathrm{mlU} / \mathrm{mL}$ versus 10.8 , SD $15.8 \mathrm{mIU} / \mathrm{mL}, P=0.003$ ) compared to those that did not require ROP treatment (Table 2). EPO levels on postnatal day 7 were nonlinearly correlated with ROP that required treatment (Fig. 2e and Table 3 ). 
Table 1. Descriptive data for infants with ROP that required or did not require treatment

\begin{tabular}{|c|c|c|c|c|}
\hline Descriptive data & Total $(n=78)$ & No ROP treatment required $(n=56)$ & ROP treatment required $(n=22)$ & $p$ value $^{a}$ \\
\hline Male & $43(55.1 \%)$ & $30(53.6 \%)$ & $13(59.1 \%)$ & 0.85 \\
\hline Birth weight $(\mathrm{g})$ & $\begin{array}{l}797(223) \\
780(415 ; 1260)\end{array}$ & $\begin{array}{l}852(222) \\
840(455 ; 1260)\end{array}$ & $\begin{array}{l}658(160) \\
635(415 ; 960)\end{array}$ & $<0.001$ \\
\hline Bronchopulmonary dysplasia & $39(50.0 \%)$ & $24(42.9 \%)$ & $15(68.2 \%)$ & 0.077 \\
\hline Necrotizing enterocolitis & $5(6.4 \%)$ & $1(1.8 \%)$ & $4(18.2 \%)$ & 0.041 \\
\hline Sepsis & 30 (38.5\%) & 21 (37.5\%) & 9 (40.9\%) & 0.98 \\
\hline
\end{tabular}
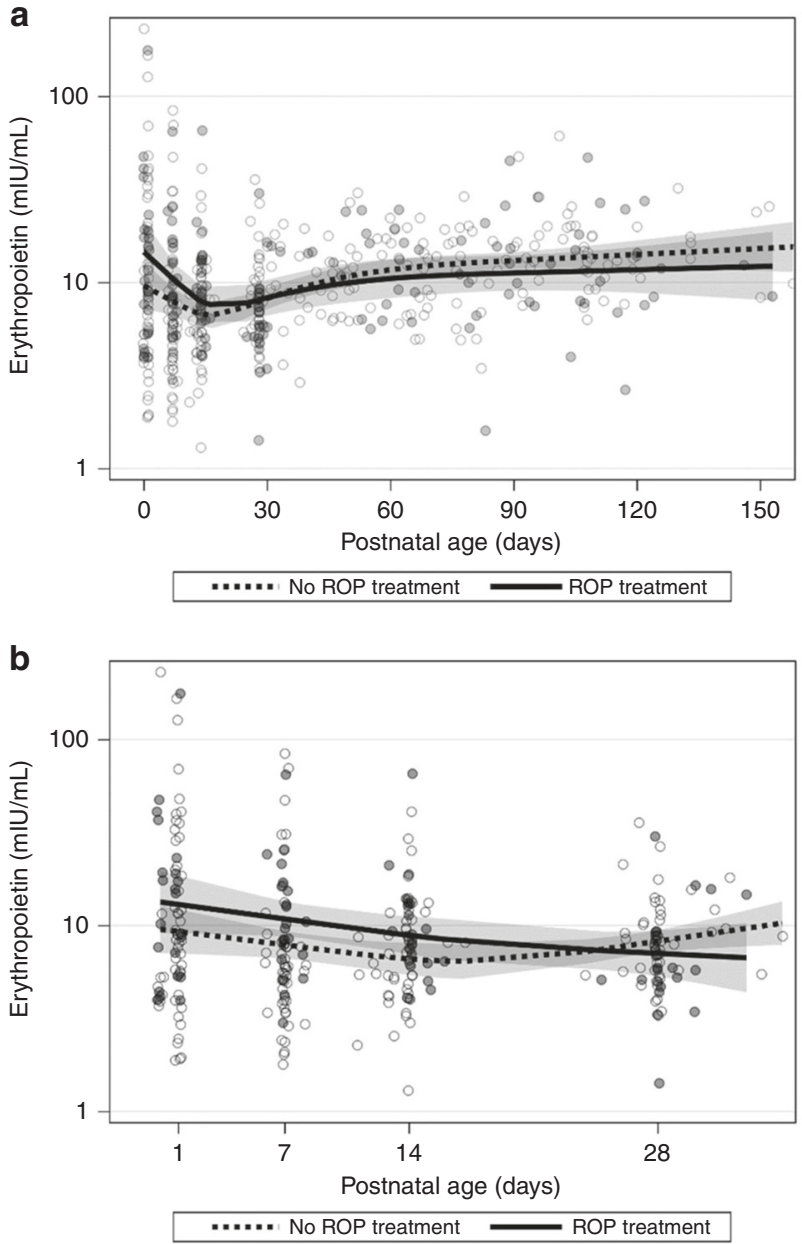

Fig. 1 Association between early erythropoietin and severe ROP requiring treatment. a Geometric mean levels of EPO from birth to 40 weeks postmenstrual age, among infants with ROP that did not require treatment (no ROP treatment, dotted line) or did require treatment (ROP treatment, solid line). $\mathbf{b}$ Geometric mean levels of EPO during the first 28 days of life; this plot is a magnification of the plot shown in a
Haemoglobin levels, anaemia and blood transfusion

Haemoglobin levels during the first week of life were significantly lower in infants that required ROP treatment than in those that did not require treatment (mean 131.8, SD $6.6 \mathrm{~g} / \mathrm{L}$ versus 136.4, SD 7.1 $\mathrm{g} / \mathrm{L}, P=0.018$ ). Accordingly, infants that required ROP treatment also developed anaemia more frequently than those that did not require treatment, during the first postnatal week $(42.9 \%$ versus $8.0 \%, P=0.003)$ and during the second postnatal week $(40.9 \%$ versus $6.3 \%, P=0.002$ ). All infants except two received at least one blood transfusion during the first four weeks of life $(76 / 78$, $97 \%)$. The mean amount of blood transfused per day was significantly higher during the four first weeks of life in infants that required ROP treatment compared to those that did not require ROP treatment (Table 2). In Fig. 2, we present the probability of anaemia (a), the mean haemoglobin levels (b) and the probability for blood transfused (c) during the first 4 weeks of life in infants that required ROP treatment compared to those that did not require treatment.

Logistic regression analysis, before and after adjusting for GA at birth

On postnatal day 7, when EPO levels was within $20 \mathrm{mIU} / \mathrm{mL}$, higher level of EPO is associated with higher risk of severe ROP requiring treatment (for each unit of EPO increases, odds ratio (OR) 1.21 (95\% Cl: 1.07-1.36), $P=0.0017)$. However, when the EPO level was above $20 \mathrm{mlU} / \mathrm{mL}$ ), the association of EPO level with severe ROP is not significant (OR 0.94 (95\% Cl: 0.87-1.01), $P=0.090$; Fig. 2e and Table 3). After adjusting for GA, EPO levels on day 7 remained a significant predictor of severe ROP (OR $1.14(95 \% \mathrm{Cl}$ : 1.00-1.30), $P=0.047$ ). On postnatal day 28, as EPO levels increased up to $10 \mathrm{mlU} / \mathrm{mL}$, the risk of severe ROP decreased (OR 0.75 (95\% Cl: 0.58-0.97), $P=0.031)$. However, after adjusting for GA at birth, the significance of the latter relationship did not persist (Table 3). Additional risk factors that remained significant after adjusting for GA at birth were; anaemia during the first and second weeks of life and the amount of blood transfused, up to $\leq 4 \mathrm{ml} /$ day, during the third and fourth weeks of life (for statistical details, see Table 3 ).

Multivariable effects of anaemia and EPO on ROP treatment Figure $2 \mathrm{~d}$ shows the relationship between EPO levels on postnatal day 7 and anaemia on days 0-7 in infants with ROP that did or did not require ROP treatment. Within the groups of infants with or without anaemia, the EPO levels were not significantly different between patients that required or did not require ROP treatment (mean 8.5 SD 4.9 versus mean 10.4 SD 16.4, respectively, $P=0.14$, for no anaemia; mean 21.6 SD 17.1 


\begin{tabular}{|c|c|c|c|c|}
\hline \multicolumn{5}{|l|}{ Erythropoietin (m/U/mL) } \\
\hline Day 7 & $\begin{array}{l}11.7(15.1) \\
6.6(1.8 ; 84.3)\end{array}$ & $\begin{array}{l}10.8(15.8) \\
6.0(1.8 ; 84.3)\end{array}$ & $\begin{array}{l}14.2(13.2) \\
10.2(3.0 ; 64.7)\end{array}$ & 0.003 \\
\hline Day 28 & $\begin{array}{l}9.12(6.04) \\
7.46(1.42 ; 35.81)\end{array}$ & $\begin{array}{l}9.53(5.95) \\
8.23(3.29 ; 35.81)\end{array}$ & $\begin{array}{l}8.19(6.30) \\
5.87(1.42 ; 30.13)\end{array}$ & 0.098 \\
\hline \multicolumn{5}{|l|}{ Haemoglobin $(\mathrm{g} / \mathrm{L})$} \\
\hline Day 0-1 & $\begin{array}{l}139.3(14.4) \\
137.0(108.8 ; 195.3)\end{array}$ & $\begin{array}{l}140.5(15.0) \\
138.6(108.8 ; 195.3)\end{array}$ & $\begin{array}{l}136.4(12.9) \\
134.0(119.0 ; 169.3)\end{array}$ & 0.13 \\
\hline Day $15-28$ & $\begin{array}{l}124.3(9.5) \\
123.7(89.0 ; 144.0)\end{array}$ & $\begin{array}{l}123.2(10.3) \\
123.2(89.0 ; 144.0)\end{array}$ & $\begin{array}{l}126.5(7.2) \\
124.5(115.4 ; 143.2\end{array}$ & 0.30 \\
\hline \multicolumn{5}{|l|}{ Anaemia (any day) } \\
\hline Day $0-1, n(\%)$ & $6(8.1 \%)$ & $5(9.6 \%)$ & $1(4.5 \%)$ & 0.84 \\
\hline Day 0-7, n (\%) & $13(18.3 \%)$ & $4(8.0 \%)$ & 9 (42.9\%) & 0.003 \\
\hline Day 8-14, n (\%) & 12 (17.1\%) & $3(6.3 \%)$ & 9 (40.9\%) & 0.002 \\
\hline Day $15-28, n(\%)$ & $37(57.8 \%)$ & $24(55.8 \%)$ & $13(61.9 \%)$ & 0.85 \\
\hline \multicolumn{5}{|c|}{ Blood transfusion ( $\mathrm{mL} /$ day) } \\
\hline Day 0-1 & $\begin{array}{l}6.50(5.13) \\
6.00(0.00 ; 25.00)\end{array}$ & $\begin{array}{l}6.68(5.37) \\
6.00(0.00 ; 25.00)\end{array}$ & $\begin{array}{l}6.07(4.58) \\
6.00(0.00 ; 14.00)\end{array}$ & 0.87 \\
\hline Day 0-7 & $\begin{array}{l}4.02(2.34) \\
4.00(0.00 ; 10.33)\end{array}$ & $\begin{array}{l}3.53(2.17) \\
3.33(0.00 ; 7.67)\end{array}$ & $\begin{array}{l}5.23(2.34) \\
5.67(1.29 ; 10.33)\end{array}$ & 0.011 \\
\hline
\end{tabular}

versus mean 18.1 SD 12.0, respectively, $P=0.82$, for anaemia). However, overall, the EPO levels could distinguish between patients with ROP that did and did not require treatment (mean 14.2, SD 13.2 versus mean 10.8 SD 15.8 , respectively, $P=0.0030$ ) (Fig. 2d). These findings were confirmed when the GA-adjusted multivariable logistic model was also adjusted for early anaemia (days 0-7), Supplemental Table S1. In this analysis, we found that EPO levels on day 7 were no longer significantly associated with ROP that required treatment (OR 1.04 (95\% Cl: 0.89-1.22), $P$ $=0.64$, for EPO values $\leq 20 \mathrm{mIU} / \mathrm{mL}$ ). This result reflected the strong correlation between EPO levels and anaemia (mean $\mathrm{EPO}=10.0$, SD 14.7, without anaemia versus mean $\mathrm{EPO}=20.6$, SD 15.3 with anaemia, $P<0.001)$.

\section{DISCUSSION}

To our knowledge, this study was the first to explore longitudinal EPO levels relative to haemoglobin levels/anaemia and ROP development in preterm infants. Our analyses revealed that mean EPO levels were significantly higher on postnatal day 7 in infants treated for severe ROP compared to infants that did not require ROP treatment. When we adjusted the model for GA at birth, the higher EPO levels up to $20 \mathrm{mIU} / \mathrm{mL}$ on postnatal day 7 remained significantly associated with severe ROP. In a multivariable logistic model, we adjusted for anaemia during days $0-7$, in addition to the GA adjustment. With this model, the association between EPO on day 7 and severe ROP was no longer present. However, anaemia during the first week of life remained an independent risk factor for ROP that required treatment. These results suggested that the associations described previously between elevated EPO levels and increased risk of severe ROP might have reflected the EPO response to anaemia, and that early anaemia was the actual risk factor. Holm et al. ${ }^{13,14}$ reported that serum EPO levels in the highest quartile on postnatal day 14 were associated with severe ROP that required treatment. Yang et al. ${ }^{15}$ reported that significantly lower EPO levels on postnatal day 28 were associated with infants that developed any degree of ROP. However, neither of those studies addressed the correlation between EPO levels and anaemia. Previous studies have reported that early anaemia was a risk factor for ROP. ${ }^{30,31}$ Reducing early anaemia in preterm infants might reduce the risk of ROP development. However, the role and impact of early anaemia needs to be evaluated further in clinical studies. 

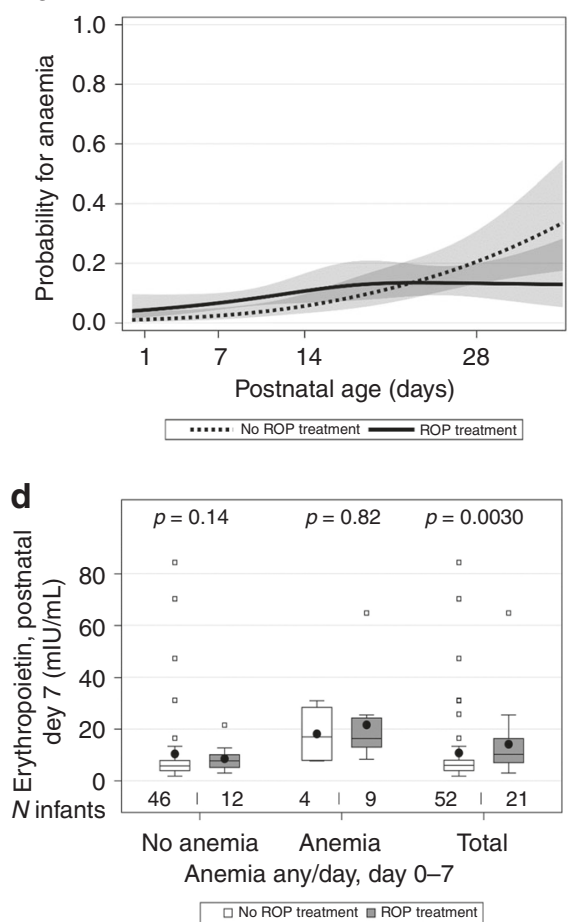

b

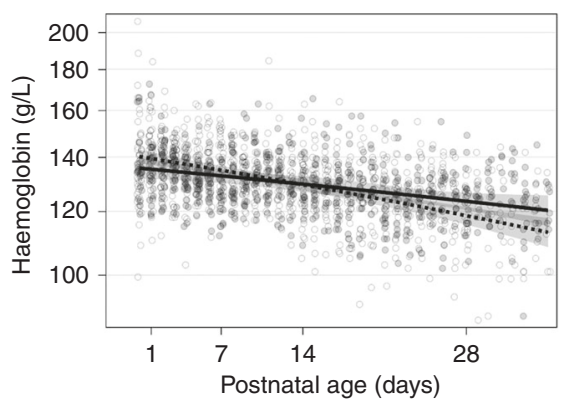

...... No ROP treatment

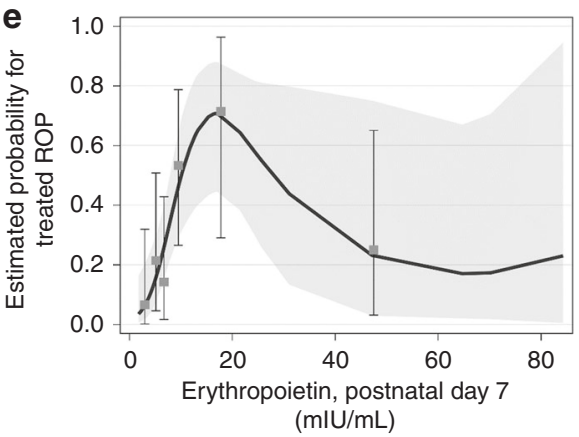

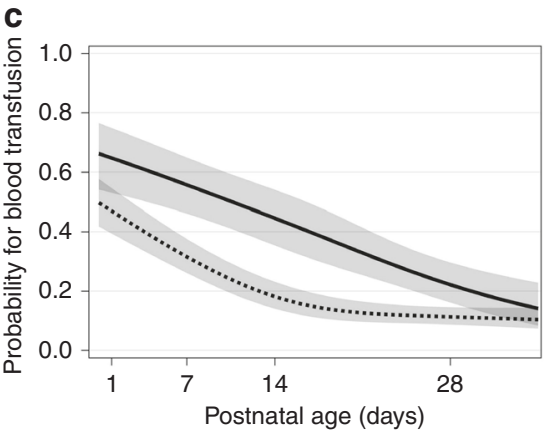

...... No ROP treatment

Fig. 2 Association between anaemia and EPO levels in infants with ROP. a Probability of developing anaemia during the first 28 days of life, among infants that did not require ROP treatment (no ROP treatment, dotted line) and those that did require ROP treatment (ROP treatment, solid line). $\mathbf{b}$ Mean levels of haemoglobin during the first 28 days of life among infants that did not require ROP treatment and infants that require ROP treatment. c Probability for blood transfusion during the first 28 days of life, among infants that did not require ROP treatment and infants that require ROP treatment. d Mean EPO levels on postnatal day 7 for infants with and without anaemia during days $0-7$ and with ROP that required ROP treatment (grey boxes) or did not require ROP treatment (white boxes). e The estimated probability and the observed proportion with $95 \% \mathrm{Cl}$ of developing ROP that required treatment, for different EPO levels on day 7

Interestingly, we found that as EPO levels on postnatal day 7 increased up to $20 \mathrm{mIU} / \mathrm{mL}$, the risk of developing severe ROP increased. However, EPO levels above $20 \mathrm{mIU} / \mathrm{mL}$ showed no association. This nonlinear correlation between EPO levels and severe ROP development might have led to at least some of the inconsistencies observed among different studies that investigated rhEPO treatment as a risk factor for ROP. Various rhEPO doses have been evaluated, from $200^{32}$ to $5000 \mathrm{U} / \mathrm{kg} /$ week; $^{33}$ however, to our knowledge, serum EPO levels were not measured in any of the trials that evaluated rhEPO treatment outcomes. We hypothesized that the nonlinear correlation between EPO and the risk of severe ROP might have reflected an inadequate effect of low EPO doses ( $\leq 20 \mathrm{mlU} / \mathrm{mL}$ ) on anaemia, which increased the risk for treated ROP; and an adequate effect of high EPO doses ( $>20$ $\mathrm{mIU} / \mathrm{mL}$ ) on anaemia, which might reduce the risk of treated ROP.

In the present study, we showed that elevated serum EPO levels did not increase the risk of developing severe ROP. However, the question remains of how rhEPO treatment impacts ROP development. Both theoretical and animal models have indicated that the timing of rhEPO treatment was important in the pathophysiology of ROP. In a mouse model of ROP, induced with oxygen administration, local retinal EPO levels were suppressed in the first phase of ROP. In this model, early supplementation with exogenous EPO in phase 1 improved retinal neural function and prevented retinal ischaemia in phase 1. Accordingly, retinal neovascularization was suppressed in phase 2 ROP. $^{34,35}$ In the same mouse model, later rhEPO, given in phase 2 of ROP, worsened retinal neovascularization; furthermore, when EPO was suppressed with antibodies, retinal neovascularisation was suppressed. ${ }^{34}$ Studies have shown inconsistent results on whether rhEPO treatment in preterm infants increased the risk of ROP. ${ }^{16-23}$

The relationship between ROP development and anaemia seems to be a complex issue, regardless of whether anaemia is corrected with blood transfusions or rhEPO. Haemoglobin in neonates consists predominantly of foetal haemoglobin at birth, which gradually declines replaced by adult haemoglobin. With blood transfusions the ratio of foetal versus adult haemoglobin is radical and abruptly changed in the preterm infant which have been hypothesized to be unfavourable for ROP development. ${ }^{36}$ There is an urgent need for studies to clarify the relationship between timing and volume of blood transfusions and ROP development. Presently, two large ongoing clinical trials might shed further light on rhEPO treatment for anaemia of prematurity and neonatal morbidities.

The main strength of this study was its longitudinal design, with weekly collections of data and serum EPO levels over several months. The main limitation of this study was the small number of infants with severe ROP that required treatment, which limited the statistical power of the analyses.

\section{CONCLUSION}

In summary, this cohort study demonstrated that an elevated serum EPO level was not a risk factor for ROP that required treatment, after adjusting for GA at birth and anaemia. However, anaemia during first postnatal week remained an independent significant risk factor for ROP that required treatment. Reducing early anaemia in preterm infants might reduce the risk of developing ROP. 


\begin{tabular}{|c|c|c|c|c|c|c|}
\hline Predictors & Value & \multicolumn{2}{|c|}{ Unadjusted logistic regression } & \multicolumn{3}{|c|}{ Gestational age-adjusted logistic regression } \\
\hline \multicolumn{7}{|c|}{ Erythropoietin $(\mathrm{m} / \mathrm{U} / \mathrm{mL})$} \\
\hline Days $0-1$ & 1 unit increase & $1.00(0.99-1.01)$ & 0.96 & $1.00(0.98-1.01)$ & 0.67 & 0.82 \\
\hline Day 14 & 1 unit increase & $1.04(0.98-1.10)$ & 0.18 & $1.02(0.97-1.08)$ & 0.42 & 0.79 \\
\hline \multirow[t]{2}{*}{ Day 28} & 1 unit increase for values $\leq 10$ & $0.75(0.58-0.97)$ & 0.031 & $0.78(0.58-1.04)$ & 0.089 & 0.80 \\
\hline & 1 unit increase for values $>10$ & $1.06(0.94-1.20)$ & 0.35 & $1.08(0.95-1.23)$ & 0.22 & \\
\hline \multicolumn{7}{|c|}{ Haemoglobin $(g / L)$} \\
\hline Days $0-1$ & 1 unit increase & $0.98(0.94-1.02)$ & 0.28 & $1.03(0.98-1.08)$ & 0.25 & 0.83 \\
\hline \multicolumn{7}{|c|}{ Anaemia (any day) } \\
\hline Days $0-1$ & Yes versus no & $0.45(0.05-4.07)$ & 0.48 & $0.18(0.01-2.97)$ & 0.23 & 0.83 \\
\hline Days 0-7 & Yes versus no & $8.62(2.26-32.88)$ & 0.002 & $7.31(1.62-32.92)$ & 0.010 & 0.84 \\
\hline Days 8-14 & Yes versus no & $10.38(2.45-44.05)$ & 0.002 & $5.41(1.16-25.27)$ & 0.032 & 0.81 \\
\hline Days $15-28$ & Yes versus no & $1.29(0.44-3.74)$ & 0.64 & $0.91(0.27-3.10)$ & 0.89 & 0.77 \\
\hline \multicolumn{7}{|c|}{ Blood transfusion ( $\mathrm{mL} /$ day) } \\
\hline Days 0-1 & 1 unit increase & $0.98(0.88-1.08)$ & 0.64 & $0.97(0.85-1.09)$ & 0.58 & 0.82 \\
\hline Days 0-7 & 1 unit increase & $1.41(1.10-1.82)$ & 0.008 & $1.29(0.96-1.72)$ & 0.094 & 0.82 \\
\hline Days 8-14 & 1 unit increase & $1.32(1.05-1.67)$ & 0.018 & $1.09(0.83-1.45)$ & 0.53 & 0.80 \\
\hline Days 15-28 & 1 unit increase for values $\leq 4$ & $2.98(1.68-5.29)$ & $<0.001$ & $2.22(1.18-4.19)$ & 0.013 & 0.84 \\
\hline
\end{tabular}

\section{ACKNOWLEDGEMENTS}

We thank all participants, the study team led by Carola Pfeiffer-Mosesson, Anne Rosenqvist, Mary Gustafsson, Svetlana Najm, Clara Mannheimer and Anna Thurfjell for very valuable help with retrieving data for the study. This study was supported by grants provided by the Swedish Research Council (DNR\# 2011-2432), Gothenburg County Council (ALFGBG-426531), De Blindas Vänner, Kronprinsessan Margaretas Arbetsnämnd för synskadade, Stiftelsen Handlanden Herman Svenssons fond för blinda och synsvaga, Carmen och Bertil Regnérs Stiftelse, Cronqvists stiftelse, NIH EY024864, EY017017, P01 HD18655, Lowy Medical Research Institute.

\section{AUTHORS CONTRIBUTION}

P.L. and A.H. designed the study, participated in the acquisition, analyses and interpretation of data, drafted the article and critically revised and approved the final draft. A.P. participated in the analyses and interpretation of data, and critically revised and approved the final draft. G.H., K.S. and L.E.H.S. critically revised and approved the final draft.

\section{ADDITIONAL INFORMATION}

The online version of this article (https://doi.org/10.1038/s41390-018-0186-6) contains supplementary material, which is available to authorized users.

Competing interests: The authors declare no competing interests.
Ethics statement: This study was approved by the Regional Ethics Board, Gothenburg (Dnr 303-11) (Clinical trial NCT02760472).

Publisher's note: Springer Nature remains neutral with regard to jurisdictional claims in published maps and institutional affiliations.

\section{REFERENCES}

1. Jelkmann, W. E., Fandrey, J., Frede, S. \& Pagel, H. Inhibition of erythropoietin production by cytokines. Implications for the anemia involved in inflammatory states. Ann. NY Acad. Sci. 718, 300-309 (1994). discussion9-11.

2. Arcasoy, M. O. The non-haematopoietic biological effects of erythropoietin. Br. J. Haematol. 141, 14-31 (2008).

3. Bunn, H. F. Erythropoietin. Cold Spring Harb. Perspect. Med. 3, a011619 (2013).

4. Jopling, J., Henry, E., Wiedmeier, S. E. \& Christensen, R. D. Reference ranges for hematocrit and blood hemoglobin concentration during the neonatal period: data from a multihospital health care system. Pediatrics 123, e333-e337 (2009).

5. Bain, A. \& Blackburn, S. Issues in transfusing preterm infants in the NICU. J. Perinat. Neonatal Nurs. 18, 170-182 (2004). quiz83-4.

6. Maier, R. F. et al. Changing practices of red blood cell transfusions in infants with birth weights less than 1000 g. J. Pediatr. 136, 220-224 (2000).

7. Bishara, N. \& Ohls, R. K. Current controversies in the management of the anemia of prematurity. Semin. Perinatol. 33, 29-34 (2009). 
Erythropoietin serum levels, versus anaemia as risk factors for severe...

$P$ Lundgren et al.

282

8. Strauss, R. G. Anaemia of prematurity: pathophysiology and treatment. Blood. Rev. 24, 221-225 (2010)

9. Blencowe, H., Lawn, J. E., Vazquez, T., Fielder, A. \& Gilbert, C. Preterm-associated visual impairment and estimates of retinopathy of prematurity at regional and global levels for 2010. Pediatr. Res. 74(Suppl. 1), 35-49 (2013).

10. Aiello, L. P. et al. Suppression of retinal neovascularization in vivo by inhibition of vascular endothelial growth factor (VEGF) using soluble VEGF-receptor chimeric proteins. Proc. Natl. Acad. Sci. USA 92, 10457-10461 (1995).

11. Giannantonio, C. et al. Analysis of risk factors for progression to treatmentrequiring $\mathrm{ROP}$ in a single neonatal intensive care unit: is the exposure time relevant? J. Matern. Fetal Neonatal Med. 25, 471-477 (2012).

12. Sato, T., Kusaka, S., Shimojo, H. \& Fujikado, T. Vitreous levels of erythropoietin and vascular endothelial growth factor in eyes with retinopathy of prematurity. Ophthalmology 116, 1599-1603 (2009).

13. Holm, M. et al. Systemic endogenous erythropoietin and associated disorders in extremely preterm newborns. Arch. Dis. Child Fetal Neonatal Ed. 101, F458-F463 (2016).

14. Holm, M. et al. Systemic inflammation-associated proteins and retinopathy of prematurity in infants born before the 28th week of gestation. Invest. Ophthalmol. Vis. Sci. 58, 6419-6428 (2017).

15. Yang, X., Ze, B., Dai, Y., Zhu, L. \& Chen, C. The alteration and significance of erythropoietin serum levels in preterm infants with retinopathy of prematurity. Am. J. Perinatol. 34, 1020-1025 (2017).

16. Suk, K. K. et al. Human recombinant erythropoietin and the incidence of retinopathy of prematurity: a multiple regression model. J. AAPOS 12, 233-238 (2008).

17. Brown, M. S., Baron, A. E., France, E. K. \& Hamman, R. F. Association between higher cumulative doses of recombinant erythropoietin and risk for retinopathy of prematurity. J. AAPOS 10, 143-149 (2006).

18. Shah, N. et al. The effect of recombinant human erythropoietin on the development of retinopathy of prematurity. Am. J. Perinatol. 27, 67-71 (2010).

19. Schneider, J. K., Gardner, D. K. \& Cordero, L. Use of recombinant human erythropoietin and risk of severe retinopathy in extremely low-birth-weight infants. Pharmacotherapy 28, 1335-1340 (2008).

20. Ohlsson, A. \& Aher, S. M. Early erythropoiesis-stimulating agents in preterm or low birth weight infants. Cochrane Database Syst. Rev. 11, Cd004863 (2017).

21. Aher, S. M. \& Ohlsson, A. Early versus late erythropoietin for preventing red blood cell transfusion in preterm and/or low birth weight infants. Cochrane Database Syst. Rev. 10, Cd004865 (2012).
22. Aher, S. M. \& Ohlsson, A. Late erythropoietin for preventing red blood cell transfusion in preterm and/or low birth weight infants. Cochrane Database Syst. Rev. Cd004868 4 (2014).

23. Chou, H. H., Chung, M. Y., Zhou, X. G. \& Lin, H. C. Early erythropoietin administration does not increase the risk of retinopathy in preterm infants. Pediatr. Neonatol. 58, 48-56 (2017).

24. Chen, J. \& Smith, L. E. A double-edged sword: erythropoietin eyed in retinopathy of prematurity. J. AAPOS 12, 221-222 (2008).

25. Najm, S. et al. Effects of a lipid emulsion containing fish oil on polyunsaturated fatty acid profiles, growth and morbidities in extremely premature infants: a randomized controlled trial. Clin. Nutr. ESPEN 20, 17-23 (2017).

26. Kliegman, R. M. \& Walsh, M. C. Neonatal necrotizing enterocolitis: pathogenesis, classification, and spectrum of illness. Curr. Probl. Pediatr. 17, 213-288 (1987).

27. International Committee for the Classification of Retinopathy of Prematurity. The International Classification of Retinopathy of Prematurity revisited. Arch. Ophthalmol. (Chic., III: 1960) 123, 991-999 (2005).

28. Early Treatment For Retinopathy Of Prematurity Cooperative Group. Revised indications for the treatment of retinopathy of prematurity: results of the early treatment for retinopathy of prematurity randomized trial. Arch. Ophthalmol. (Chic., III: 1960) 121, 1684-1694 (2003).

29. Altman, D. G., Vergouwe, Y., Royston, P. \& Moons, K. G. Prognosis and prognostic research: validating a prognostic model. BMJ (Clin. Res. Ed.) 338, b605 (2009).

30. Lundgren, P. et al. Duration of anaemia during the first week of life is an independent risk factor for retinopathy of prematurity. Acta Paediatr. (Oslo, Nor.: 1992) 107, 759-766 (2017).

31. Banerjee, J. et al. Haemoglobin level at birth is associated with short term outcomes and mortality in preterm infants. BMC Med. 13, 16 (2015).

32. Shannon, K. M. et al. Recombinant human erythropoietin in the anemia of prematurity: results of a placebo-controlled pilot study. J. Pediatr. 118, 949-955 (1991).

33. Gumy-Pause, F. et al. Stepping up versus standard doses of erythropoietin in preterm infants: a randomized controlled trial. Pediatr. Hematol. Oncol. 22, 667-678 (2005).

34. Chen, J., Connor, K. M., Aderman, C. M. \& Smith, L. E. Erythropoietin deficiency decreases vascular stability in mice. J. Clin. Invest. 118, 526-533 (2008).

35. Mowat, F. M. et al. Endogenous erythropoietin protects neuroretinal function in ischemic retinopathy. Am. J. Pathol. 180, 1726-1739 (2012).

36. Stutchfield, C. J., Jain, A., Odd, D., Williams, C. \& Markham, R. Foetal haemoglobin, blood transfusion, and retinopathy of prematurity in very preterm infants: a pilot prospective cohort study. Eye 31, 1451-1455 (2017). 